\title{
artigo
}

Fontes da Silva, V.R.; Lyra da Silva, R.C.; Marta, C.B.; Garcia A.S.; Vicentini, S.C.; Lyra da Silva, C.R.

Análise bibliométrica da produção científica sobre Coronavírus e Covid-19

DOI: https://doi.org/10.36489/saudecoletiva.2020v10i53p2356-2369

\section{Análise bibliométrica da produção científica sobre Coronavírus e Covid-19}

Bibliometric analysis of scientific production on coronavirus and Covid-19

Análisis bibliométrico de la producción científica en Coronavirus y Covid-19

\section{RESUMO}

Objetivo: analisar a produção intelectual sobre Coronavírus e COVID-19, disponibilizada na base Web of Science. Método: estudo bibliométrico que abordou a produção registrada eletronicamente, de 2016 a 2020, com análise estatística descritiva. Resultados: recuperou-se 1697 documentos publicados em 500 periódicos, A média da produção por autoria foi de 1,652730752. A categoria Enfermagem contribuiu com quatro (0,238\%) documentos, ficando com a Virologia a maior contribuição 320 (19\%), Os EUA lideraram com 479 artigos (28,5\%). Conclusão: os dados apresentados demonstram o alto nível de interesse da comunidade científico pelo assunto. Foi possível identificar 82 autores compondo um grupo de Elite de Autores, cuja produtividade foi de $66,1 \%$. Os Estados Unidos, China e Arábia Saudita são os países que mais formaram Redes de Colaboração.

DESCRIPTORS: COVID-19; Coronavírus; Bibliometria; Infectologia; Medicina; Virologia.

\section{ABSTRACT}

Objective: to analyze the intellectual production on Coronavirus and COVID-19, made available on the Web of Science base. Method: bibliometric study that addressed the production recorded electronically, from 2016 to 2020, with descriptive statistical analysis. Results: 1697 documents published in 500 journals, The average production per author was 1.652730752. The Nursing category contributed four $(0.238 \%)$ documents, with Virology making the largest contribution $320(19 \%)$, The USA led with 479 articles (28.5\%). Conclusion: the data presented demonstrate the high level of interest of the scientific community on the subject. It was possible to identify 82 authors composing an Elite group of Authors, whose productivity was $66.1 \%$. The United States, China and Saudi Arabia are the countries that most formed Collaboration Networks.

DESCRIPTORES: COVID-19; Coronavirus; Bibliometry; Infectious diseases; Medicine; Virology.

\section{RESUMEN}

Objetivo: analizar la producción intelectual sobre Coronavirus y COVID-19, disponible en la base de datos de Web of Science. Método: estudio bibliométrico que abordó la producción registrada electrónicamente, de 2016 a 2020, con análisis estadístico descriptivo. Resultados: se recuperaron 1697 documentos publicados en 500 revistas y la producción promedio por autor fue de 1.652730752. La categoría de Enfermería contribuyó con cuatro (0.238\%) documentos, siendo Virology la mayor contribución $320(19 \%)$, EE. UU. Lideró con 479 artículos (28.5\%). Conclusión: los datos presentados demuestran el alto nivel de interés de la comunidad científica en el tema. Fue posible identificar 82 autores que componen un grupo Elite de Autores, cuya productividad fue del 66,1\%. Estados Unidos, China y Arabia Saudita son los países que más formaron Redes de Colaboración.

DESCRITORES: COVID-19; Coronavirus; Bibliometría; Enfermedades infecciosas; Medicina; Virología.

RECEBIDO EM: 07/04/2020 APROVADO EM: 08/04/2020

\section{Viviane Reis Fontes da Silva}

Enfermeira. Mestra e Doutoranda do Programa de Pós-Graduação em Enfermagem e Biociências da Universidade Federal do Rio de Janeiro - UNIRIO. Rio de Janeiro - RJ, Brasil. ORCID https://orcid.org/0000-0003-4174-8544.

\section{Cezar Cheng}

Engenheiro da Informação. Engenheiro do Instituto Nacional do Câncer. Doutorando Programa de Pós-Graduação em Enfermagem e Biociências. Rio de Janeiro, RJ, Brasil. ORCID https://orcid.org/0000-0001-8831-3885.

\section{Roberto Carlos Lyra da Silva}

Enfermeiro. Doutor em Enfermagem, Coordenador do Programa Pós-Graduação em Enfermagem e Biociências da Universidade Federal do Estado do Rio de Janeiro - UNIRIO. Rio de Janeiro - RJ, Brasil. ORCID https://orcid.org/0000-0002-2125-1437. 


\section{Cristiano Bertolossi Marta}

Enfermeiro. Doutor em Enfermagem. Docente do Departamento de Fundamentos de Enfermagem da Faculdade de Enfermagem da Universidade do Estado do Rio de Janeiro - UERJ e Diretor de Pesquisa da Universidade Veiga de Almeida - UVA. Rio de Janeiro - RJ, Brasil. ORCID https://orcid.org/0000-0002-0635-7970.

\section{Andrea dos Santos Garcia}

Enfermeira. Mestra e Doutoranda do Programa de Pós-graduação em Enfermagem e Biociências da Universidade Federal do Rio de Janeiro - UNIRIO. Rio de Janeiro - RJ, Brasil. ORCID https://orcid.org/0000-0002-2125-1437.

\section{Solange Campos Vicentini}

Médica. Doutoranda do Programa de Pós-graduação em Enfermagem e Biociências da Universidade Federal do Rio de Janeiro - UNIRIO. Docente do Instituto Biomédico da Universidade Federal do Estado do Rio de Janeiro - UNIRO. ORCID https://orcid. org/0000-0002-4202-7514.

\section{Carlos Roberto Lyra da Silva}

Enfermeiro. Doutor em Enfermagem, docente dos Programas de Mestrado e Doutorado em Enfermagem e Biociências da Universidade Federal do Estado do Rio de Janeiro (UNIRIO). Rio de Janeiro, RJ, Brasil. ORCID https://orcid.org/0000-0002-4327-6272.

\section{INTRODUÇÃO}

$\mathbf{0}$ s Coronavírus compóem uma grande família de vírus, conhecidos desde meados da década de 1960. Podem causar desde um resfriado comum até síndromes respiratórias graves, como a Síndrome Respiratória Aguda Grave (SARS - Severe Acute Respiratory Syndrome) e a Síndrome Respiratória do Oriente Médio (MERS - Middle East Respiratory Syndrome). Trata-se de uma família de vírus que, majoritariamente, afeta animais, sendo que em humanos são conhecidas sete variedades, quatro já tinham sido detectadas no Brasil e foram responsáveis por infecções respiratórias de pouca importância ${ }^{(1)}$.

Os casos identificados em 2020 estão relacionados a uma nova variante do Coronavírus, denominada SARS- CoV-2, responsável pela doença denominada de COVID-19 (do inglês, Coronavirus Disease-2019). É uma doença infecciosa emergente, identificada pela primeira vez na cidade chinesa de Wuhan, cujos casos iniciais foram diagnosticados em dezembro de 2019. No dia 11 de março de 2020, o Diretor Geral da Organização Mundial de Saúde (OMS), Sr, Tedros Adhanom Ghebreyesus declara pandemia causada por essa nova variante ${ }^{(1)}$.

Situações como essa, em que o mun- do inteiro se encontra em alerta para combater essa doença, a sociedade civil precisa confiar naqueles que detêm o poder político, mas principalmente, naqueles que produzem conhecimento científico acessível de forma rápida e qualificada, sendo a Rede Mundial de Computadores, a fonte preferida de profissionais de saúde, estudantes, pesquisadores, bem como da sociedade de uma forma geral, em que pese o fato de que, nem sempre, esta fonte pode ser considerada confiável.

Em decorrência dos fatores intervenientes da pandemia do novo Coronavírus estarem no plano da multidisciplinaridade, uma vez que envolve aspectos das ciências básicas e das aplicadas, passando pela Virologia, Microbiologia, Infectologia, Epidemiologia, Estatística, Enfermagem, Psicologia, Sociologia, Biologia entre outras, observa-se que pesquisadores das mais diferentes áreas de conhecimento, encontram-se focados em suas pesquisas em busca de uma tratamento eficaz e de uma vacina capaz de imunizar o ser humano, portanto, o estado da arte de tais pesquisas poderá se beneficiar em decorrência de estudos bibliométricos como este.

Vivenciamos um século cuja informação é publicada e disseminada em grande escala e velocidade sem precedentes, tornando cada vez mais fácil o processo de incorporação por parte dos pesquisadores ${ }^{(2)}$, portanto, é fundamental que se tenha o mínimo de habilidade para selecionar as melhores bases de indexação e repositórios, prospectar, filtrar e selecionar as informações mais relevantes para suas pesquisas, e é justamente neste nesse contexto que os estudos bibliométricos se apresentam como fontes de informação para diferentes áreas de pesquisa, e que são capazes de subsidiar a evolução tecnológica e científica ${ }^{(3)}$, não obstante, pela sua característica de mapear a produção científica $^{(4)}$.

A análise bibliométrica, que compreende a aplicação da estatística à bibliografia, possui três leis classicamente reconhecidas: Lei Bradford (lei de dispersão do conhecimento científico), Lei de Lotka (lei de produtividade de autores) e Lei de Zipf (frequência de palavras). Vale destacar que a principal diferença entre bibliografia e bibliometria é que esta última utiliza, principalmente, métodos quantitativos do que discursivos, o que confere maior objetividade na avaliação da produção científica(5), não se preocupando somente com o aspecto quantitativo, mas também em verificar a relevância e o impacto de autores, periódicos, instituições, grupos ou países nas mais diversas áreas ${ }^{(4,5)}$.

Os estudos bibliométricos estão embasados em um conjunto de leis e 
princípios empíricos, provenientes da ciência da informação, cujo objetivo é investigar os aspectos quantitativos da produção, da disseminação e do uso da informação disponível e registrada, contribuindo, dessa forma, para a avaliação do estado atual da ciência, assim como do gerenciamento da pesquisa ${ }^{(5-7)}$.

Este estudo justifica-se por pesquisar as características da produção científica sobre Coronavírus e COVID-19, contribuindo para a comunidade científica interessada em estudar este vírus, pois mostrará a distribuição da produção por ano, área geográfica, área de conhecimento, identificar os periódicos mais devotados ao assunto, os autores mais produtivos, entre outros aspectos. A questão desta proposição é: A indexação de documentos sobre Coronavírus e COVID-19 na base Web of Science, publicados nos últimos cinco anos, obedece às leis e princípios bibliométricos de Lotka e de Bradford?

Com a finalidade de responder a esta questão por meio da utilização de métricas bibliométricas, tem-se como objetivo analisar a produção de artigos sobre Coronavírus e COVID-19, disponibilizada na base Web of Science.

\section{METODOLOGIA}

Trata-se de um estudo bibliométrico que abordou a produção/disseminação e o uso da informação registrada eletronicamente em base de dados internacional, publicada no período de 2016 a 2020, período este baseado no índice de Price $(5,6)$ que representa a proporção em $50 \%$ do consumo de informação com idade entre zero e cinco anos. O princípio da bibliometria compreende ao uso de indicadores confiáveis, que podem ser definidos como parâmetros utilizados em processos de avaliação(5). O material de análise foi limitado a documentos indexados na base Web of Science, para tanto, utilizou-se como critério de inclusão, todos os tipos de documentos publicados entre 2016 a 2020, não havendo critérios de exclusão.
Trata-se de um estudo bibliométrico que abordou

a produção/

disseminação e o

uso da informação

registrada

eletronicamente

em base de dados

internacional,

publicada no

período de 2016

a 2020, período

este baseado no

índice de Price $(5,6)$

que representa a

proporção em $\mathbf{5 0 \%}$

do consumo de

informação com

idade entre zero e

cinco anos.
As buscas foram realizadas na base de dados da Web of Science no mês de abril de 2020, utilizando-se os descritores [Coronavírus] e [COVID-19] e com filtro "tópico". O acesso se deu via Portal de Periódicos da CAPES, usando as credenciais do pesquisador na Rede Cafe.

A escolha da base se deve por sua aceitação na comunidade científica mundial, sobretudo para a área da saúde, a qual disponibiliza resumos e citações de literatura científica revisada por pares, além de oferecer uma visão mais abrangente sobre a produção de pesquisa do mundo. Para a realização da etapa das análises bibliométricas, utilizou-se a interface gráfica do software VOSViewers, e a ferramenta Microsoft Excel $2017^{\circ}$. A análise descritiva calculou a média, mediana e o desvio padrão. Os resultados obtidos são apresentados na sequência.

\section{RESULTADOS}

A busca retornou com 1.697 documentos, dos quais, 1.017 (59,9\%) receberam citações que totalizaram 9.372 com média de 9,372 citações por documento. A primeira análise destinou-se a levantar a quantidade de documentos por ano, bem como identificar em termos percentuais a produção científica. Em 2020 foram publicados 563 (33,53\%) documentos, 2019286 (17,03\%), 2018253 (15,06\%), 2017 251 (14,94\%) e em 2016326 (19,41\%).

Foram contabilizados 6.793 pesquisadores entre autores e coautores. A média de produtividade foi pouco mais que 1,65 , com mediana 1 e desvio padrão 1,845943562. O autor/ coautor mais producente publicou 34 artigos (2\%), enquanto a maioria absoluta, $4.956(72,9 \%)$ autores/coautores publicaram somente um único artigo. Os dez mais produtivos são apresentados na Tabela 1 . A equação $\mathrm{X} \alpha^{*} \mathrm{Y}=\mathrm{C}$, proposta na Lei de Lotka foi aplicada no conjunto total de autores/coautores, onde $\mathrm{X}=$ número de documentos 
Tabela 1. Os dez autores/coautores mais produtivos. Rio de Janeiro, RJ, Brasil, 2020.

\section{ORDEM DE SÉRIE}

1

2

3

4

5

6

7

8

9

10
AUTORES

Perlman, S

Baric, RS

Drosten, C

Yuen, KY

Shi, ZI

Memish, ZA

Haagmans, BL

Mahase, E

Gerber, Si

Al-Tawfiq, JA

\section{QUANTIDADE DE ARTIGOS}

34

30

29

27

25

25

25

24

22

22

Nota: ${ }^{*}$ Equivalente à ordem de série.
Gráfico 1. Constante de Lotka, Rio de Janeiro, RJ, Brasil, 2020

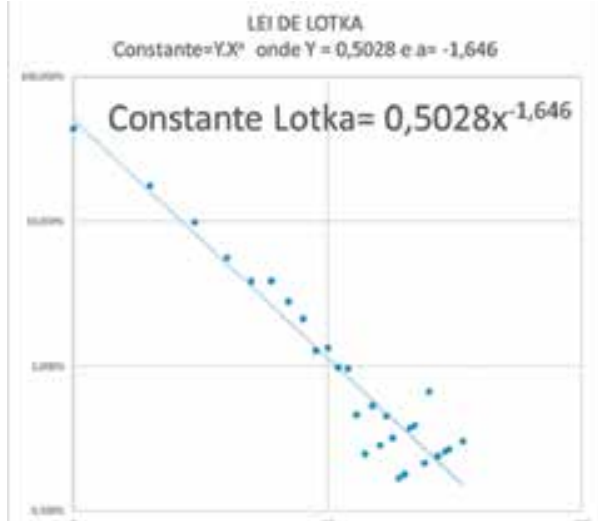

Figura 1. Mapa de colaboração autoral por país. Rio de Janeiro, RJ, Brasil, 2018
$\%$ OF 1679

2.025

1.787

1.727

1.608

1.489

1.489

1.489

1.429

1.310

1.310

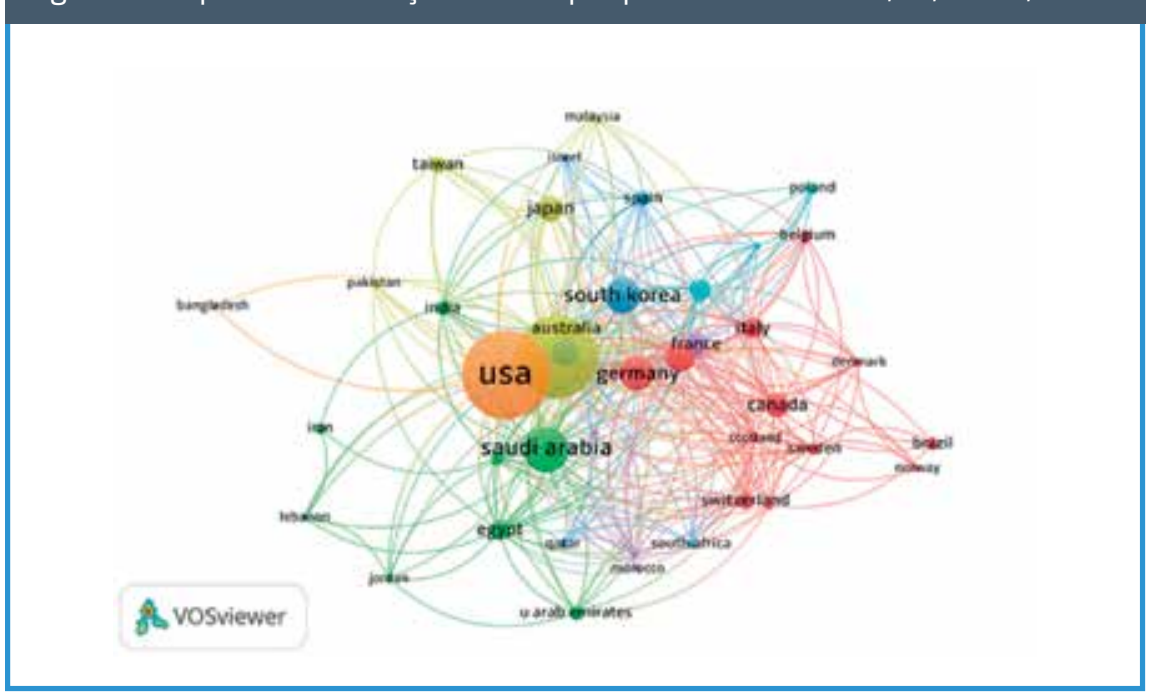

publicados, $\mathrm{Y}=$ número de autores com $\mathrm{X}$ publicações e $\mathrm{C}=$ constante. $\mathrm{O}$ Gráfico 1 representa a equação a partir da distribuição de artigos pelo número de autores, cuja constante foi de Lotka foi igual a 0,5028X-1,646.

Dos documentos recuperados, os mais frequentes foram os artigos originais 1.015 $(60,4 \%)$, seguido pelos artigos de revisão 141 (8,3\%) e editoriais 212 (12,6\%). O restante se encaixava em outras modalidades.

A distribuição geográfica das publicações recuperadas contou com a colaboração de 100 países, A liderança absoluta ficou com os Estados Unidos da América (EUA) com 479 documentos (28,5\%), seguidos de perto pela China com 431 (26,6\%), o Brasil ocupa a 21 a posição com 23 (1,3\%). Os outros 97 países publicaram juntos 933 artigos $(44,6)$ de toda produção.

A análise geográfica de coautoria considerou os países com no mínimo 5 produções e 10 citações por documento, representando, portanto, 41 (41\%), menos da metade dos países com produção, enquanto $24(24 \%)$ países publicaram apenas um único documento. A Figura 1 representa essa distribuição, destacando-se a Bulgária, Chile, Perú, Sérvia, Senegal, Venezuela, Ulcrânia e Zambia, cada qual contribuindo com apenas $0,06 \%$ da produção. Neste cenário, o Brasil forma um hub de colaboração com apenas cinco países: Itália, Inglaterra, Canadá, Suécia e Suíça.

O idioma das publicações está concentrado na língua inglesa, com $1.657(98,6)$ documentos, seguidos pelo Alemão seis $(0,3 \%)$ e, apenas um $(0,06 \%)$ documento em Português.

Foram recuperadas 1.815 instituições envolvidas na produção dos documentos recuperados, das quais, 346 (19,06\%) são universidades e $80(4,4 \%)$ hospitais, A Tabela 2 destaca as 10 instituições mais producentes.

Dentre as categorias, apenas oito das 103 recuperadas, produziram na ordem de uma centena, Virologia 320 (19,05\%), Doenças Infecciosas 297 (17,68\%), Microbiologia 200 (11,91\%), Medicina geral interna $173(10,3 \%)$, Imunologia 156 (9,29\%), Veterinária 145 (8.63\%) e 
Tabela 2. Quantidade da Produção por instituição. Rio de Janeiro, RJ, Brasil, 2020.

$\begin{array}{cccc}\mathbf{R}^{*} & \text { INSTITUIÇÃO } & \text { PRODUÇÃO } & \text { \% OF 1679 } \\ 1 & \text { University of hong kong } & 76 & 4,527 \\ 2 & \text { Chinese academy of sciences } & 62 & 3,693 \\ 3 & \text { King saud bin abdulaziz university for } & 42 & 2,501 \\ 4 & \text { health sciences } & 39 & 2,323 \\ 5 & \text { Ministry of health saudi arabia } & 39 & 2,323 \\ 6 & \text { University of north carolina } & 38 & 2,263 \\ 7 & \text { National institutes of health nih usa } & 38 & 2,263 \\ 8 & \text { Seoul national university snu } & 38 & 2,263 \\ 9 & \text { University of north carolina chapel hill } & 37 & 2,204 \\ 10 & \text { Johns hopkins university } & 36 & 2,144\end{array}$

Nota: *Equivalente à ordem de série.

\section{Tabela 3. Tabela Clássica de Bradford. Rio de Janeiro, RJ, Brasil, 2020.}

$\mathbf{R}^{*}$

2

3

4

5

6

7

8

9

10

11

12

13

14

15

16

17

18

19

20

21

22

23

24

25
NO PERIÓDICOS

\section{NO ARTIGOS}

\section{B}

70

66

40

32

31

31

29

28

27

25

24

22

20

19

18

17

16

15

14

13

12

11

10

9

8

\section{ACUM.}

\section{C}

1

2

3

4

5

6

7

7

8

9

10

12

13

14

15

17

18

19

20

24

25

29

37

38

45

51
LOG (C)

ACUM. B

$A^{*} B$

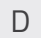

70

70

0,30103

0,47712

136

66

176

0,60206

0,69897

0,77815

0,8451

0,90309

0,95424

1

1,07918

1,11394

1,14613

1,17609

1,23045

1,25527

1,27875

1,30103

1,38021

1,39794

1,4624

1,5682

1,57978

1,65321

1,70757
208

239

270

299

327

354

379

403

425

445

464

482

499

515

530

544

557

569

580

590

599

607
Saúde ocupacional ambiental pública 128 (7,62\%). A Enfermagem contribuiu com 4 (0,23\%) documento. Os 1.697 documentos encontrados estão distribuídos em 500 periódicos, com média de 3,39 artigos por periódico. O número de periódicos que publicou um único documento foi de 291 (58,2\%).

Os dez periódicos com mais documentos publicados foram: Journal of Virology 70 (4,16\%), British Medical Jornal 66 (3,93\%), Viruses Basel 40 (2,38\%), Emerging Microbes Infections 32 (1,9\%), Emerging Infectious Diseases 31 (1,84\%), Lancet 31 (1.84\%), Virology 29 (1,72), Scientific Reports 28 (1.66\%), Eurosurveillance $27(1,6 \%)$ e Nature 25 (1,48\%). A média, mediana e desvio padrão da produção foram, sucessivamente: 3,394, 1 e 6,435339244.

A Tabela 3 apresenta a Tabela de Bradford com distribuição dos periódicos e sua produção, de acordo com as três zonas, aqui denominadas de zonas de Bradford, cujo enunciado proposto pelo teórico confere com a organização dos periódicos científicos em ordem de produtividade decrescente de documentos sobre um determinado assunto, seu conjunto poderá ser dividido em um núcleo de periódicos mais particularmente dedicado ao assunto e vários grupos ou zonas que devem conter o mesmo número de documentos que o núcleo, então o número de periódicos no núcleo e nas zonas sucessivas, será a proporção 1:n:n2:n3 ...

Para fins de distribuição do núcleo e das zonas, neste estudo, utilizou-se o valor proposto na teoria, assim, o núcleo e cada uma das duas Zonas, contabilizou, aproximadamente, $1 / 3$ do total de documentos produzidos no recorte temporal analisado, ou seja, aproximadamente 566 documentos no núcleo e em cada zona. O Multiplicador de Bradford ( $\mathrm{Bm})$, foi também calculado para mostrar que o número de periódicos contidos numa zona em relação à subsequente, deve se manter oscilando, no máximo, 0,7 de diferença. A Tabela 4 mostra todos esses valores. 


$\begin{array}{ccccccc}26 & 9 & 7 & 60 & 1,77815 & 614 & 63 \\ 27 & 8 & 6 & 68 & 1,83251 & 620 & 48 \\ 28 & 13 & 5 & 81 & 1,90849 & 625 & 65 \\ 29 & 22 & 4 & 103 & 2,01284 & 629 & 88 \\ 30 & 32 & 3 & 135 & 2,13033 & 632 & 96 \\ 31 & 74 & 2 & 209 & 2,32015 & 634 & 148 \\ 32 & 291 & 1 & 500 & 2,69897 & 635 & 291\end{array}$

Nota: *Equivalente à ordem de série.

\section{Tabela 4. Zonas de Bradford. Rio de Janeiro, RJ, Brasil, 2020}

\section{ZONAS}

Núcleo

Zona 1

Zona 2

\section{CONTAGEM DE PERIÓDICOS}

20

83

397

\section{PROPORÇÃO}

\section{1}

n

$n * 2$
$\mathrm{BM}^{*}$

4,15

4,783133
Nota: Bm* Multiplicador de Bradford

\section{DISCUSSÃO}

Após estas análises, foi possível comprovar que o corpus da pesquisa é consistente, pois a mineração dos dados permitiu a recuperação dos documentos a partir dos principais descritores, delimitando o assunto que foi o foco da pesquisa. A quantidade de 1.697 documentos em cinco anos de estudo, superou outros estudos bibliométricos na área de saúde, como por exemplo, sobre produção científica sobre assédio moral/assédio moral com 53 documentos recuperados no período de 2002 a $2012^{(8)}$ e outro sobre realidade virtual na punção venosa, que analisou os 213 documentos recuperados na mesma base de dados, de 1969 a $2018^{(9)}$. Este resultado reflete muito o interesse da comunidade científica acerca da pandemia, bem como sua emergência no cenário mundial.

Observou-se um aumento sustentável de publicações a partir de 2016, sendo o ano de 2020, mesmo ainda em curso, foi o que teve o maior número de publicações, justificando mais uma vez a emergência do assunto e a curva de interesse por parte da comunidade científica. É inegável que a vida média(5,10) dessa produção ainda não atingiu seu pico. Contudo, pelo comportamento da produção científica, esse número tende a ser superado, haja vista que a consulta na Web of Science ocorreu em abril de 2020.

O percentual de artigos que receberam as 9.372 (59,9\%) citações, permitiu determinar o quão extenso são os canais de transferência desse conhecimento, seu consumo e utilidade, ção sobre Coronavírus e COVID-19 pelos pesquisadores. Permite ainda afirmar com segurança que o ritmo do desbastamento ou a substituição do conhecimento científico sobre esse assunto ainda está longe de ser iniciado, da mesma forma que o fator de envelhecimento/obsolescência, uma vez que não houve perda superior a $50 \%$ nas citações e na atualidade da informaçãa ${ }^{(10)}$.

Aplicando-se a lei de Lotka, verifica-se que de fato é possível identificar uma relação inversamente proporcional entre a quantidade de autores e artigos produzidos. Não obstante, a constante encontrada no Gráfico 2, confirma o postulado que representa o pensamento 'muitos com pouco e poucos com muito ${ }^{(7-10)}$, uma vez que, quanto maior o número de artigos produzidos, menor é a quantidade de autores.

Ao aplicar a Lei do Elitismo de Price ${ }^{(10)}$, foi possível identificar o Grupo de Elite de autores, tendo em vista que raiz quadrada do total de autores foi 82 , total de autores que representa a elite da área estudada, já que sua produção representa $66,1 \%$ dos documentos recuperados, ultrapassando em muito o mínimo de 50\% Proposto na lei do Elitismo, em que peso o fato de que o índice de produtividade tenha sido muito baixo 1,65, em grande parte por consequência do número excessivo de autores ocasionais ${ }^{(10)}$. já que eles estão sendo a fonte de informa-
A análise de coautoria possibilitou verificar a força de colaboração científica entre aqueles autores/coautores que produziram no mínimo 5 documentos com no mínimo 10 citações, estando sua maioria compondo o Grupo de Elite. A análise de coautoria é um importante indicador bibliométrico, sendo um dos atributos mais pesquisados na utilização da Análise de Redes Sociais (ARS), pois, proporciona ao pesquisador uma visão ampla dos colégios invisíveis nos quais os vértices da pesquisa estão imersos, além de uma série de outras constatações quanto às relações de união no âmbito científico ${ }^{(8-10)}$.

Neste aspecto, quando comparamos a média de coautoria com as instituiçõos e países de origem, é possível constatar que as Redes Sociais no âmbito científico estão globalizadas entre autores, filiação e países, mais uma vez, lideradas pelos Estados Unidos da América e países orientais, em particular, China e Arábia Saudita, seguidos pelo Reino Unido e Alemanha. Destaca-se a 21 a posição do Brasil, único representante da América Latina. O idioma das publicações foi dominado pelo Inglês, cuja dominância pode ser facilmente explicada, por ser o inglês um idioma universal para a ciência. Esta constatação permite inferir com segurança que o nível de interação internacional dos pesquisadores brasileiros ainda está em fase de consolidação.

Aplicando a Lei de Bradford para verificar o comportamento da distribuição/ dispersão dos periódicos, na Tabela 3, foi possível identificar, a partir do Multiplicador de Bradford (Bm), uma constante, já que a variação entre o $\mathrm{Bm}$ das zonas se manteve dentro da oscilação permitida ${ }^{(5-9)}, 0,7$, demonstrando distribuição muito próxima da ideal de - I:n:n2, conforme determina a Lei. O núcleo comportou 18 periódicos, sendo estes os mais devotados ao assunto ${ }^{(9,10)}$.

\section{CONCLUSÃO}

Considera-se que títulos e resumos de artigos são relevantes como representativos na produção científica acerca do tema. Os resultados apresentam alta produção de estudos, distribuídos pelas mais variadas áreas de conhecimento, no entanto, restringindo-se à Saúde. Por se tratar de um assunto 
bastante atual, quando se considera o recorte temporal, é razoável afirmar que quantidade de documentos recuperados é bastante relevante quando comparados a outros estudos bibliométricos da área de saúde.

A Bibliometria contribuiu para estudar o registro da produção, A estruturação de bases de dados facilitou a obtenção dos mesmos e os softwares disponíveis no mercado, por sua vez, agilizam o tratamento e a análise quantitativa de tais dados.

Os pesquisadores podem beneficiar-se das técnicas de análise bibliométricas, não apenas em uma abordagem quantitativa, mas também qualitativa, pois os números auxiliam na leitura aproximada da realidade e, com a inclusão de estudos mais aprofundados, a riqueza das análises torna-se mais representativa. Portanto, os estudos bibliométricos podem contribuir para a visualização das conexões entre informações de diversas áreas do conhecimento,

Quanto aos autores, evidenciou-se a quantidade expressiva de autores por artigo e, quanto aos mais produtivos, o estudo destacou o periódico que veiculou suas pesquisas, a instituição ao qual estão vinculados e sua localiza- ção geográfica, neste particular, fica evidente que $\mathrm{o}$ assunto tem despertado o interesse de pesquisadores ao ponto de constituírem redes de colaboração interpaíses e interinstituições, bem como um Grupo de Elite.

Muito embora baseada em fatos empíricos, as leis de Bradford e Lotka, conseguiram neste estudo, confirmar possíveis hipóteses teóricas de que o núcleo de periódicos é formado pelos mais devotados e, portanto, mais produtivos, não obstante, revelou que quanto maior for a especificidade do tema estudado, maior será a possibilidade de identificação de grupos de Elite de autores.

Como foi utilizada a formulação clássica da Lei, era previsível que o ajuste não fosse perfeito, uma vez que n variáveis influenciam na distribuição empírica da literatura, tais quais não são captadas pela fórmula clássica de Bradford, e representam o maior desafio para os estudiosos contemporâneos de sua formulação matemática. O núcleo apontado a partir da aplicação da Lei reconheceu perfeitamente um importante aspecto relativo ao comportamento acadêmico científico sobre Coronavírus e COVID-19, ou seja, quando há a evidência de que determinados periódicos são ou terão reconhecimento no meio, mais e mais publicações são a ele direcionadas, para tanto, seria importante verificar o aspecto qualitativo desses periódicos, com base nos indicadores de fator de impacto, estes seriam os mais confiáveis.

Outro aspecto importante e que precisa ser considerado, refere-se à institucionalização cognitiva das áreas de conhecimento de domínio dos artigos e periódicos recuperados, que pôde ser identificada pelo uso dos dois descritores utilizados. Em que pese a padronização gramatical dos descritores por um vocabulário controlado - Descritores em Ciências da Saúde (DeCS), ainda assim, a realização de inferências não confiáveis pode se configurar em uma limitação deste estudo.

Como contribuição, os resultados do estudo e seu desenho metodológico poderão servir de base para outras proposições, preenchendo assim, uma lacuna existente no rol de pesquisas bibliométricas em saúde, principalmente na enfermagem, como o compromisso de consolidar seu corpo de conhecimento, desenvolvendo pesquisas que possam contribuir simultaneamente para o crescimento e consolidação desse saber.

\section{REFERÊNCIAS}

1. Góes LGB, Zerbinati RM, Tateno AF, et al. Epidemiologia típica de infecções por vírus respiratórios em uma favela brasileira. Journal Med, Virol. [Internet]. 2019;1(6) [acesso em 4 abr 2020]. Disponivel: https://doi,org/10,1002/jmv,25636.

2. Pimenta AA, Portela ARMR, Oliveira CB, Ribeiro RM. A bibliometria nas pesquisas acadêmicas. Scientia [Internet] 2017; 4(7):1(13) [acesso em 4 abr 2020]. Disponivel em: https://bit,ly/ 2VqvJ25.

3. Mugnaini R, Jannuzzi PM, Quoniam L. Indicadores bibliométricos da produção científica brasileira: uma análise a partir da base Pascal. Ci Inf [Internet]. 2004;33(2):123-31[acesso em 4 abr 2020], Disponível em: http://www,scielo,br/pdf/ci/v33n2/ a13v33n2.

4. Aria M, Cuccurullo C. Bibliometrix: An R-tool for comprehensive science mapping analysis, J Informetr [Internet]. 2017;11(4):959-75[acesso em 4 abr 2020]. Disponível em: https://www.sciencedirect.com/science/article/abs/pii/ S1751157717300500.

5. Araújo CAA, Bibliometria: evolução histórica e questões atuais, Em Questão, [Internet]. 2006;15(4) [acesso em 1 mai 2018]. Disponível em: http://seer.ufrgs.br/index.php/EmQuestao/\%20article/view/16.

6. Barreto $\mathrm{ML}$, The challenge of assessing the impact of science beyond bibliometrics, Rev, Saúde Pública, [Internet] 2013;47(4) [acesso em 1 mai 2018]. Disponivel: http://dx,doi,org/10,1590/ S0034-8910,2013047005073,

7. Medeiros JMG, Vitoriano MAV. A evolução da bibliometria e sua interdisciplinaridade na produção científica brasileira. Rev Digit Biblioteconomia Ciênc Info, [Internet]. 2015;13(3) [acesso em 1 mai 2018]. Disponível: https://periodicos.sbu.unicamp.br/ ojs/index.php/rdbci/article/view/8635791.

8. Costa, ICP, Costa, SFG da, Andrade, CG de, Oliveira, RC de, Abrão, FM da Silva, Silva, CRL da. Produção científica sobre assédio moral/assédio moral em dissertações e teses no cenário brasileiro. Rev. Esc. Enferm. USP [Internet]. 2015; 49 (2): 0267-0276 [acesso em 4 abr 2020]. Disponível em: http://www.scielo.br/scielo.php?script=sci_arttext\&pid=S0080-62342015000200267\&lng=en. https://doi. org/10.1590/S0080-623420150000200012.

9. Da Silva, VRF, Meireles, IB, Cheng, C, Silva, RCL, Silva, CRL, Santiago, LC. Análise bibliométrica de artigos sobre cateterização venosa periférica em pediatria. Cogitare Enfermagem [Internet]. 2019;24. [acesso em 4 abr 2020]. Disponivel em: https://revistas.ufpr.br/cogitare/article/view/59232.

10. Urbizagástegui, AR. Obsolescência da literatura sobre a Lei de Lotka. Data Grama Zero: revista de Ciência da Informação [Internet]. 2009;10(1). [acesso em 4 abr 2020]. Disponível em: http://www.dgz.org.br/ago12/F_I_art.htm. 